\title{
Perspectives
}

\section{Metabolic and Genetic Characterization of Prediabetic States Sequence of Events Leading to Non-Insulin-dependent Diabetes Mellitus}

\author{
Henning Beck-Nielsen and Leif C. Groop \\ Diabetes Research Center, Medical Endocrinological Department M, Odense University Hospital, DK-5000 Odense, Denmark; \\ and Department of Endocrinology, University of Lund, Malmö General Hospital, Malmö, S-21401 Sweden
}

Non-insulin-dependent diabetes mellitus (NIDDM) ${ }^{1}$ is phenotypically heterogenous and may therefore not be explained by one simple pathophysiological mechanism. Despite the heterogenous nature of the disease, the major form as discussed in this review is linked to overweight, arterial hypertension, dyslipidemia, and macroangiopathy (coronary heart disease), all conditions which become more common with aging (1). This form of NIDDM seems to be growing worldwide in the footsteps of Westernized life style. Around 3\% in the Western world suffers from the disease, but in subjects $60 \mathrm{yr}$ and older, the prevalence is as high as $10-20 \%(2)$. In certain ethnic populations as the Pima Indians and the Nauruans the prevalence approaches $40 \%$ (2). Therefore, NIDDM imposes an enormous burden on the health care system all over the world.

In general, the goals of treatment are achieved in only a small subset of patients, but nevertheless progression of macroangiopathy is a more severe problem than worsening of glycemia. The overall mortality rate in patients with NIDDM is two to three times higher than in control subjects, primarily due to excess mortality of coronary heart disease (2). Of note, $\sim 40 \%$ of patients already suffer from clinical macroangiopathy at diagnosis, indicating that these complications may not be secondary to the diabetic state itself, but rather a part of the NIDDM syndrome, also referred to as Syndrome $\mathrm{X}$ or "The Insulin Resistance Syndrome"' (1).

Resistance to the action of insulin on glucose metabolism in skeletal muscle has been proposed as the inherited basis for this syndrome and since insulin resistance is observed several decades before the onset of hyperglycemia an early detection can open the prospect of intervention in order to prevent the disease process (4-7).

The present review discusses the metabolic and genetic background of NIDDM together with the current knowledge of the sequence of events leading to NIDDM. Based on that a new

Address correspondence to Henning Beck-Nielsen, M.D., Dr. Sci., Professor of Medicine, Medical Endocrinological Department M, Odense University Hospital, Kloevervaenget 6, DK-5000 Odense, Denmark. 1994.

Received for publication 2 June 1994 and in revised form 5 August

1. Abbreviations used in this paper: HGP, hepatic glucose production; IGT, impaired glucose tolerance; NGT, normal glucose tolerance; NIDDM, non-insulin-dependent diabetes mellitus.

J. Clin. Invest.

(C) The American Society for Clinical Investigation, Inc. 0021-9738/94/11/1714/08 \$2.00

Volume 94, November 1994, 1714-1721 staging system is proposed in order to improve treatment and favor prevention.

\section{The inheritance of NIDDM}

The prevalence of NIDDM varies greatly between different parts of the world. The question therefore rises, are these ethnical differences due to different genetic background or to differences in environmental factors? The genetic contribution is quite obvious in some of these populations. There is a higher prevalence of NIDDM among pure Nauruans than in Nauruans with foreign genetic admixture. Furthermore, there is a higher prevalence of NIDDM in full-blood North Dakota Indians aged over $35 \mathrm{yr}$ than in persons with less than one-half Indian inheritance ( 22.2 vs $4.1 \%$ ). Insulin resistance in Pima Indians shows a trimodal distribution consistent with the hypothesis that insulin resistance is determined by a single gene. Similar data are so far lacking in Europid subjects. Given the heterogenous nature of the disease, it is rather likely that several genes contribute to insulin resistance in Europid subjects with NIDDM. The genetic nature of NIDDM in Europids is supported by the following investigations. The concordance rate of NIDDM in monozygotic twins has been found to be around 70-80\%, compared with $10-20 \%$ in dizygotic twins. There is a clear clustering of imparied glucose tolerance (IGT) and NIDDM in families. In offsprings and in sibs of patients with NIDDM the lifetime risk of developing NIDDM has been estimated to $\sim 40 \%$ (3). If both parents suffer from the disease the risk in insulin resistant children reaches $80 \%$ (6). Thus, NIDDM seems to be a strongly inherited disease, although the mode of inheritance is not known.

Pathophysiology of NIDDM: what is the primary inherited defect?

Although both peripheral insulin resistance and insulin deficiency contribute to hyperglycemia in NIDDM, hyperglycemia per se can further impair insulin sensitivity and beta-cell function. A vicious cycle ensues, which is able to maintain hyperglycemia. Therefore, subjects with manifest hyperglycemia usually display all defects of diabetes, i.e., insulin resistance and impaired beta-cell function. However, what comes first, insulin resistance or impaired beta-cell function?

Insulin resistance. To address this question we have studied persons at increased risk of developing NIDDM, i.e., first degree relatives of patients with $\operatorname{NIDDM}(4,5)$. In the Finnish study, the relatives could be divided into two groups based upon whether glucose tolerance was normal or impaired. As expected, patients with NIDDM and IGT were insulin resistant compared with healthy subjects, but surprisingly, relatives with normal oral glucose tolerance (NGT) also showed a significant impairment in their ability to metabolize glucose (4). In the Danish 
study lean and younger relatives were investigated. However, the results were identical confirming that even young lean first degree relatives to NIDDM patients were insulin resistant in peripheral tissues (5). Similar findings have recently been reported in offspring of Mexican Americans with NIDDM (7). The defect in insulin action was localized only to the nonoxidative pathway in skeletal muscles, i.e., glycogen synthesis. In accordance to that we found that the covalent activation of the enzyme glycogen synthase was reduced in parallel to the reduction in glycogen synthesis (5).

These results clearly indicate that $\sim 50 \%$ of first degree relatives are insulin resistant 3-4 decades before they are expected to develop Type 2 diabetes. But is insulin resistance a risk factor for progression to NIDDM? This question has been addressed in three prospective studies. The Joshlin group studied offspring of two parents with NIDDM (7). They found that insulin resistant (and glucose resistant) subjects had an $80 \%$ increased risk of developing NIDDM during a 25-yr follow up period. In accordance with this, Skarfors et al. (9) demonstrated that a low glucose clearance rate during an intravenous glucose tolerance test was an independent risk factor for developing NIDDM in elderly Swedish men (9). Similarly, in The Malmö NIDDM Prevention Study a high insulin response during an oral glucose tolerance test (indicating insulin resistance) predicted development of NIDDM in middle-aged men (10). Taken together, these results strongly indicated that peripheral insulin resistance is an inherited defect predicting development of frank diabetes mellitus.

Insulin secretion. In relatives with normal glucose tolerance (IVGT) all phases of insulin secretion estimated by hyperglycemic clamp were normal (4). This finding is in accordance with the results obtained in the prospective Joslin study (6), where both first phase and second phase responses to an IVGTT challenge were found to be increased in prediabetics with normal glucose tolerance (NGT). In fact, subjects who developed NIDDM within a 10-20-yr period tended to present higher initial insulin responses to glucose compared with the subjects who continued to be normoglycemic. However, this finding does not exclude an early beta cell defect since insulin secretion rates must be viewed in relation to the degree of insulin resistance. By doing that, even NGT relatives may present a lower than expected insulin secretion rate (our own unpublished data). Relatives with IGT were in our study always associated with impaired first phase insulin secretion and once the patients had progressed to manifest diabetes, all phases of insulin secretion became impaired. In the Swedish studies of elderly subjects a reduced first phase insulin response during IVGTT in addition to insulin resistance was found to predict development of $\operatorname{NIDDM}(9,10)$. We recently confirmed this finding in a study of elderly identical twins discordant for NIDDM, demonstrating a high concordance rate for insulin deficiancy in the nondiabetic twin (8). Altogether, these data suggest that insulin resistance alone is not enough to manifest glucose intolerance including NIDDM, an additional defect in insulin secretion is required. This defect seems to be inherited and expressed before frank hyperglycemia.

Hepatic glucose production (HGP). Basal HGP was found to be normal in normoglycemic first degree relatives to patients with NIDDM $(4,5,7)$, but also, the inhibition of hepatic glucose production by insulin seems to be unaffected at this stage (7). On the other hand, patients with impaired glucose tolerance $($ IGT + NIDDM) often demonstrate an increase in HGP after meals, indicating that the liver is resistant to the action of insulin, or that this increase in HGP is secondary to insulin deficiency (4). Enhanced rates of basal hepatic glucose production is frequently, but not always reported in patients with manifest diabetes and fasting hyperglycemia. However, a number of methodological problems make the interpretation of hepatic glucose production data difficult (11). HGP seems to be autoregulated, i.e., an increase in gluconeogenesis, as demonstrated in NIDDM subjects, is counterbalanced by a reduction in glycogenolysis whereby HGP is kept normal or near normal. It is not known whether the putative increase in gluconeogenesis in NIDDM patients is of primary origin; i.e., due to an inherited elevation of the PEPCK (phosphoenol pyruvate carboxykinase) activity or secondary to an increase in substrate supply induced by an elevation of glycolysis in muscles, although recent data favor the last hypothesis. In accordance, no data have demonstrated that insulin inhibits gluconeogenesis by inhibiting the transcription of the PEPCK gene, neither have alterations of DNA in the PEPCK gene been described. Thus, inherited defects in HGP have not been demonstrated until now.

Model for the development of NIDDM. The data presented suggest that insulin resistance, i.e., impaired stimulation of glycogen formation in skeletal muscle, represents a primary defect in the pathogenesis of NIDDM (4-7). The following model for the development of NIDDM can therefore be proposed: two genetic defects are required, most likely including several genes, one causing impaired glycogen formation in skeletal muscles, the other causing impaired beta-cell function, i.e., inability to compensate for insulin resistance. Although they act jointly to cause diabetes, insulin resistance is the trigger which increases the insulin demands thereby unmasking the defect in the beta cell. A normal beta cell may compensate for the insulin resistance by increasing the amount of insulin secreted whereas the "prediabetic" beta cell in the long term cannot, and hyperglycemia ensues. The genetic defect responsible for insulin resistance in glycogen synthesis in skeletal muscles seems to be expressed very early in life, maybe already in childhood, whereas the genetic defect responsible for the impaired beta-cell function may be expressed later on. Our current hypothesis for the pathogenesis of NIDDM is outlined in Fig. 1.

\section{Cellular defects responsible for inherited insulin resistance in skeletal muscle}

Inherited insulin resistance in NIDDM patients seems to be located mainly in the non-oxidative pathway of glucose metabolism in skeletal muscle, i.e., glycogen synthesis. When frank diabetes develops, several other metabolic defects are added to the genetic defect(s), along with reduced glucose oxidation.

Stimulation of glycogen synthase activity in skeletal muscle by insulin seems to be the result of a cascade of phosphorylation-dephosphorylation reactions (Fig. 2). The first step is insulin binding to the alpha subunit of the specific membrane receptor resulting in autophosphorylation of three tyrosine residues of the intracellular beta subunit of the receptor. The next step in activation of metabolism may be phosphorylation of the recently discovered protein IRS 1 (insulin receptor substrate 1) on tyrosine, serine, and threonine residues. IRS 1 is a docking protein for the insulin receptor but is not a kinase itself. However, IRS1 possesses abilities to bind other proteins with $\mathrm{SH}_{2}$ domains, i.e., kinases and phosphatases, whereby different insulin signals may be generated. The signal to the glycogen synthase seems to be mediated through the binding of G-proteins 


\section{Pathophysiology of NIDDM}

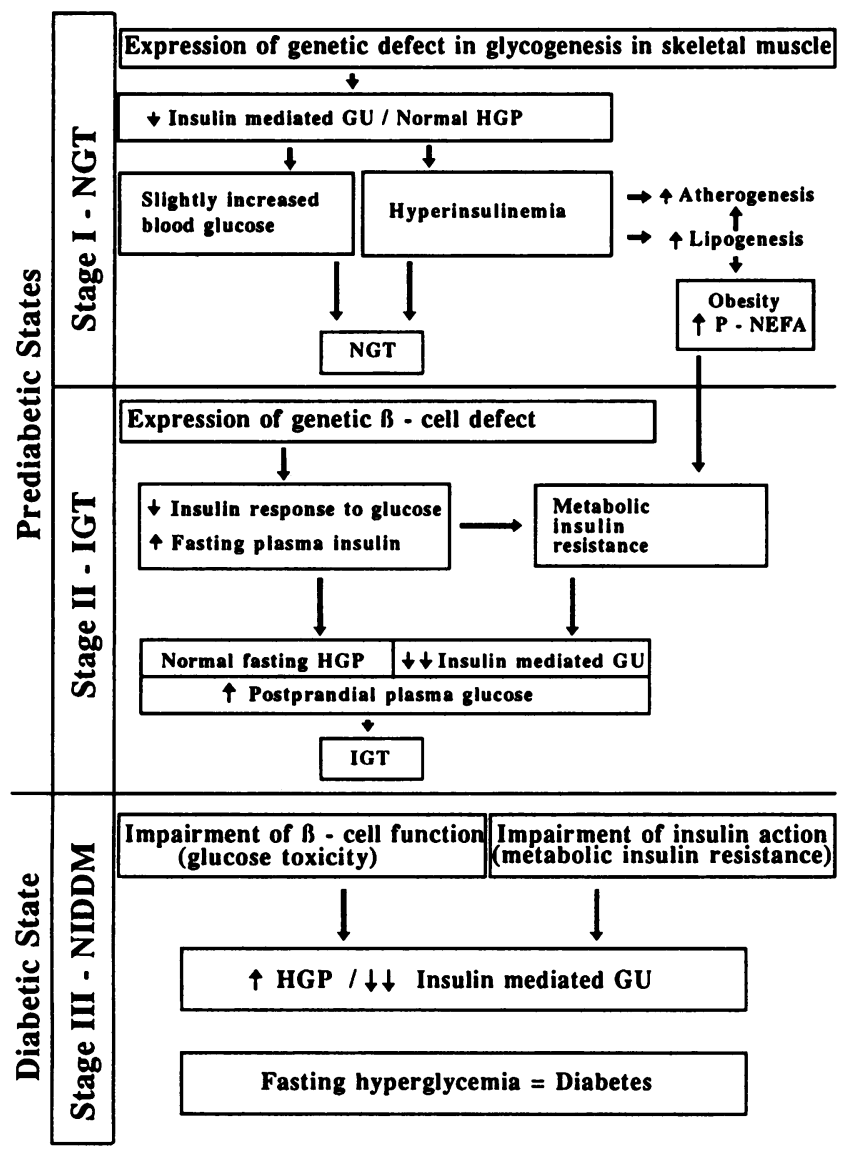

Figure 1. Hypothesis for pathophysiology of NIDDM and its prediabetic stages.

resulting in initiation of a phosphorylation-dephosphorylation cascade involving (e.g., Ras oncogenes, MAP kinases, ISPK [insulin sensitive protein kinase], and finally, phosphatases as PP1 and PP2A [protein phosphatase 1 and 2]) the enzymes responsible for dephosphorylation of glycogen synthase. This cascade mechanism may be responsible for transduction of the insulin signal from the receptor to the glycogen synthase but the involvement of intermediary pathways as Ras oncogenes and MAP kinases is still discussed. Glycogen synthase is among others rephosphorylated and inactivated by the enzyme glycogen synthase kinase 3 (GSK-3) and c-AMP-dependent kinase. IRS1 also binds a kinase, phosphatidylinositol-3-kinase (PI-3$\mathrm{K})$, which phosphorylates the insulin signal molecule, phosphatidylinositol. This mediator (PI-3-P) may be involved in translocation of GLUT4 proteins. Other systems have also been described. For example, insulin stimulates phospholipase $C_{\beta 1}$, which catalyses membrane phospholipids into two mediators, namely inositol triphosphate (InsP3), which seems to be involved in calcium signaling mechanisms and diacylglycerol (DAG), which stimulates protein kinase C (Fig. 2).

All proteins participating in insulin signaling and glucose processing in skeletal muscle may theoretically be involved in the pathophysiology of insulin resistance. Our current knowledge in this area will be reviewed below.

Insulin receptors. A reduced insulin receptor number can only explain the rightward shift in the dose-response curve but not the $V_{\max }$ defect for insulin stimulation of glycogen synthase. In spite of the fact that the insulin receptor number may be reduced even in prediabetic subjects, this defect does not seem to be the major cause of insulin resistance in skeletal muscle. Although a number of mutations have been identified in the insulin receptor gene on chromosome 19 , they can explain only some rare familial cases of NIDDM characterized by severe insulin resistance, e.g., leprachaunism, Rabdon-Mendelhalls syndrome, and Type A insulin resistance (12). The contribution of mutations in the insulin receptor gene to NIDDM is probably $<0.1 \%$.

Insulin receptor tyrosine kinase and IRS 1 . A reduced ability of insulin to stimulate the consecutive phosphorylation of the three tyrosine residues on the $\beta$ subunit has been demonstrated in obese NIDDM patients. Furthermore, transfer of phosphate from the receptor kinase to an external substrate, in this case POLY GLU 4:Tyr 1, has been found to be reduced. This finding in NIDDM, however, could be of secondary origin since it was found to be reversible at least in human fat cells (13). However, recent data from our laboratory indicate that the reduced autophosphorylation may be shown already in the prediabetic state. Mutations in the kinase domain of the insulin receptor $\beta$ subunit result in defective kinase activity, yielding the clinical phenotype of Type A insulin resistance with acanthosis nigricans. However, these rare mutations do not explain the common genetic insulin resistance in NIDDM patients in general.

The docking protein IRS1 links the tyrosine phosphorylated insulin receptor to proteins of importance for the downstream phosphorylation cascade. Studies of OB/OB mice indicated a reduced phosphorylation of IRS1 in these insulin-resistant animals. However, the expression of IRS1 in skeletal muscles from NIDDM patients was increased rather than reduced. Recently, using SSCP scanning (single stranded conformational polymorphism) two amino acid polymorphisms were described in the IRS-1 genes (14). These amino acid substitutions, which were located close to tyrosine phosphorylation motifs, were more frequent in NIDDM than in control subjects (19 vs 7\%; $P$ $=0.02$ ). It is difficult, for the moment, to interpret the role of this finding in the pathogenesis of insulin resistance in NIDDM. The insulin signaling pathway may also include phosphorylation of Ras oncogenes, and by using a subtraction library from skeletal muscle of NIDDM patients a novel Ras protein, called RAD, was found to be overexpressed in patients with NIDDM (15). It is, however, premature to conclude whether this is the cause or consequence of diabetes, since a number of other genes including IRS-1, PP1, and GS all tend to be overexpressed in skeletal muscles from NIDDM patients (16).

Glucose transporter activity. Insulin stimulates transmembrane glucose transport by translocation of intracellular glucose transporters (GLUT 4) to the cell membrane. An insulin-resistant glucose transport system seems to be a characteristic feature of skeletal muscles from obese diabetics, but the reduced transporter activity in skeletal muscle in NIDDM could be secondary to hyperglycemia, since the amount of GLUT-4 protein expressed has been shown to be normal in NIDDM and since a translocation defect has not been uniformly proven (16). No significant alterations in the genes encoding for GLUT-1 and GLUT-4 have been described in patients with NIDDM (17). In keeping with the findings with IRS-1, the GLUT-4 mRNA tends to be increased in insulin-resistant subjects (16). It is not known whether this represents a compensatory increase to 


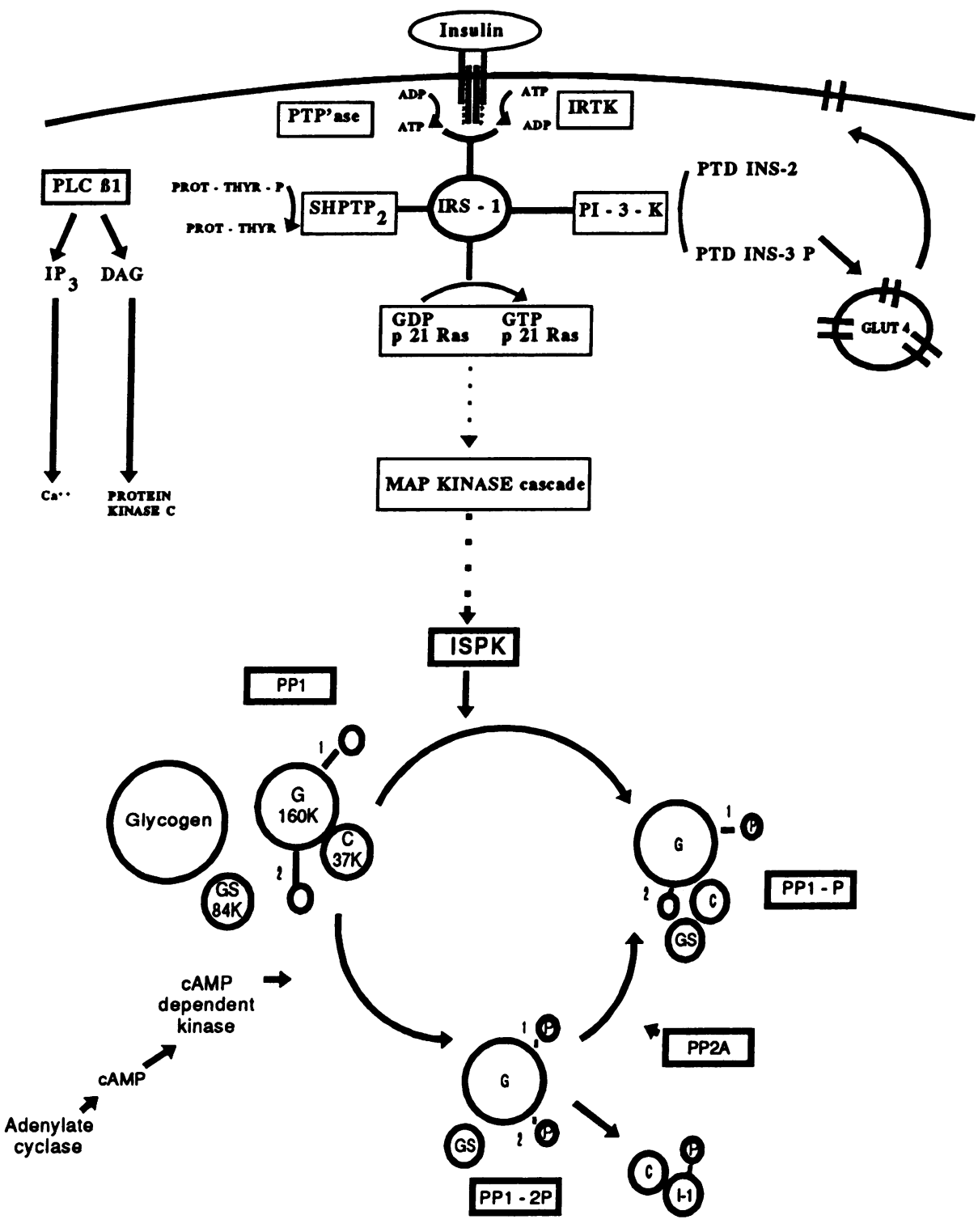

Figure 2. Model for insulin activation of glycogen synthase in skeletal muscles. Insulin activates ISPK (insulin sensitive protein kinase) through a cascade of phosphorylations and dephosphorylations in involving IRTK (insulin receptor tyrosine kinase), IRS1 (insulin receptor substrate 1), the G-protein Ras-p21, and the MAP kinase cascade. ISPK phosphorylates the G-subunit of PP1 (protein phosphatase 1 ) in position 1 , whereby the catalytic subunit $(C)$ is bound to GS (glycogen synthase). Through this binding glycogen synthase is dephosphorylated and thereby activated. cAMP-dependent kinase phosphorylates PP1 in both position 1 and 2 and thereby, the enzyme is inactivated. The $\mathrm{C}$ subunit and I-e (inhibitor 1$)$ dissociate from the $G$ subunit. PP2A, protein phosphatase $2 \mathrm{~A}$ is able to dephosphorylate PP1-2P and thereby the enzyme complex is reactivated. overcome a more distal defect in intracellular glucose metabolism.

Hexokinases. Phosphorylation of glucose to glucose-6phosphate occurs by hexokinases. It has generally been considered unlikely that defects in this phosphorylation would contribute to impaired insulin action in skeletal muscle since the $K_{\mathrm{m}}$ for glucose of HK II is in the range of 0.1 to $1.0 \mathrm{mmol} / \mathrm{liter}$, and thereby always "turned on." Preliminary studies in families of patients with NIDDM could not demonstrate linkage to the gene challenging the view that HK II would represent a major gene in the pathogenesis of NIDDM (18).

Glycolysis and glucose oxidation. We and others have recently found that the non-oxidative glycolytic flux rate in skeletal muscles in NIDDM patients is increased, which may compensate for the reduction in glucose storage. In accordance with this, the rate limiting enzyme in glycolysis, phosphofructokinase, was found to be without mutations in the coding region by SSCP scanning. Glucose oxidation in response to insulin, on the other hand, is reduced in diabetics which may be caused by a reduced activity of the enzyme pyruvate-dehydrogenase.
In prediabetic subjects, glucose oxidation is completely normal and therefore reduced glucose oxidation in NIDDM may be of secondary origin.

Glycogen synthase. In lean control subjects, insulin stimulates the fractional velocity of glycogen synthase more than twofold. However, in obese diabetic subjects, insulin is unable to increase the enzyme activity. Thus, glycogen synthase seems to be completely resistant to insulin (19). This defect is due to an abnormal covalent stimulation. The enzyme therefore seems to be partly locked into its inactive G-6-P-dependent form, but may, however, still be activated allosterically by physiological concentrations of G-6-P. In other words, an identical activity rate of glycogen synthase may be obtained in diabetic subjects as in nondiabetic subjects if the intracellular G-6-P concentration in diabetic subjects is increased sufficiently. The reduced glycogen synthase activity seems to be associated with a reduced protein phosphatase-1 activity (PP1) and an increased cAMP-dependent protein kinase activity in skeletal muscles from obese diabetic subjects compared with nondiabetic subjects. The reduced glycogen synthase activity in skeletal muscle 
in diabetics does not seem to be a phenomenon secondary to hyperglycemia, for example, since we found this enzyme defect already in normoglycemic prediabetic first-degree relatives to NIDDM patients (5). If it is induced by a signaling defect or it is a genetic defect in the molecule itself, is still a matter of debate. Recently Wells and co-workers (20) showed that insulin stimulation of glycogen synthase in cultured fibroblasts from NIDDM patients, with family clustering of the disease, was absent despite a normal activation of the insulin receptor tyrosine kinase; a finding supporting the view that a reduced glycogen synthase activity could be an inherited defect in NIDDM.

The glycogen synthase gene has therefore become an important candidate gene for NIDDM. Xbal polymorphism of the glycogen synthase has recently been associated with NIDDM in Finland, Japan, and France (21). In the Finnish diabetic patients, the XbaI polymorphism was further characterized by a strong family history of NIDDM and increased frequency of hypertension. Since the $\mathrm{XbaI}$ polymorphism is due to a single basepair change in an intron, the polymorphism can only be considered as a marker for NIDDM. The glycogen synthase gene is located on the long arm of chromosome 19(22); linkage disequilibrium with another locus in this region may explain the association found. We and others have confirmed this association using a number of other polymorphic markers in the same region. Linkage studies, however, have yielded inconclusive results, but it may be difficult to establish linkage for susceptibility genes explaining at most $20-25 \%$ of the genetic variance.

We have recently described the exon-intron structure of the GS gene and found a missense mutation in exon 11 in two families with NIDDM, hypertension, and premature arteriosclerosis (23). Screening of the promoter region with SSCP scanning has also revealed a number of polymorphisms, none of which seems to be increased in patients with NIDDM. Expression of the glycogen synthase gene is not decreased in patients with NIDDM, and no difference in the expression of the glycogen synthase gene has been observed between patients with different alleles of XbaI glycogen synthase polymorphism (24).

\section{Genetic defects in insulin secretion}

The finding of linkage between the glucokinase gene on chromosome 7 and MODY (maturity-onset diabetes in the young) was a real breakthrough in the search for diabetes-associated genes (25). Several mutations have been described in the glucokinase gene, some even occurring in the same MODY family. Of importance, the mutant enzyme in general has a lower affinity for glucose resulting in impaired glucose stimulated insulin secretion. Glucokinase mutations are observed in $\sim 60 \%$ of patients with MODY. This form of diabetes, that is further characterized by mild impairment in $\beta$-cell function, could preferentially be called glucokinase-deficient diabetes. It is questionable whether glucokinase mutations are seen in patients with classical NIDDM; the phenotype of NIDDM patients with glucokinase defects has in general been very MODY-like.

\section{Mitochondrial mutations}

Maternal transmission of NIDDM predominates, therefore mutations in mitochondrial DNA have merged as possible targets for a genetic defect leading to NIDDM. Mitochondrial DNA, which is circular and contains only $16,569 \mathrm{bp}$, is transmitted only from the mother. Mutations in mitochondrial DNA have been reported in both IDDM and NIDDM patients with deafness (26). These patients are generally characterized by some degree of beta-cell dysfunction, but it is not known how the mutation affects mitochondrial energy metabolism. Mitochondrial mutations tend to increase with age. In the same cell there can be both normal and abnormal mitochondrial DNA (heteroplasmy). Theoretically, an increasing number of mitochondrial DNA mutations could influence energy metabolism both in the beta cell and in the muscle, thereby increasing the risk for NIDDM with age.

\section{The thrifty gene theory}

Why is NIDDM increasing in individuals switching from a rural to an urban lifestyle? The thrifty gene hypothesis was put forward in 1962 by Neel (27), who proposed that for societies living in environments with unstable food supplies, maximizing storage of surplus energy would enhance the probability of survival under periods of energy deficiency. With the abundance of food typical for modern Westernized society, such a genotype might lead to marked obesity and glucose intolerance. If this hypothesis is true, it may be more economical to channel energy to fat than to glycogen in skeletal muscle. Furthermore, insulinresistant patients should have increased amounts of triglycerides in their muscles. In fact, patients with NIDDM have a four- to fivefold increase in skeletal muscle triglyceride concentrations compared with healthy control subjects, but data are lacking from insulin-resistant nondiabetic subjects. Support for the survival advantage hypothesis comes from a study of heterogeneous insulin-resistant ob and ob mice (28). When given a surplus of food, part of these animals develop hyperinsulinemia and hyperglycemia. If the thrifty gene hypothesis is correct, insulin-resistant animals should be better suited to withstand prolonged periods of starving. In fact, the insulin-resistant ob and ob mice survived longer during starvation than their insulinsensitive littermates. Interestingly, the locus for developing diabetes during fat feeding in certain mice strains was recently linked to chromosome 7 or, more precisely, to the glycogen synthase locus (29).

\section{The search for NIDDM genes}

Although there is plenty of evidence that NIDDM is inherited, there is also a number of arguments against a Mendelian mode of inheritance. The disease is more often transmitted from the mother than from the father. The penetrance of the disease is highly variable and age dependent, and dependent upon environmental factors as energy intake and exercise.

Is it a realistic task to search for the NIDDM genes? There will be nothing like a one-gene-one-disease, it is even unlikely that there will be any major gene. The genetic abnormalities will most likely be unmasked only when exposed to changes in environmental factors. There can be 4-5 genes involved that increase the susceptibility to develop diabetes under certain environmental conditions. The candidate gene approach so far has been only partially successful, but the list of candidate genes seems to be depleted soon. A random gene search has been successful for a number of monogenic diseases with well-known inheritance, but will it work for NIDDM? A conventional linkage analysis would require that the gene(s) involved would explain a major part of the disease. It requires further information about mode of inheritance and penetrance, two unknowns in the NIDDM gene equation.

There are some ways to improve the odds. If the population studied is homogenous, random markers at $10-\mathrm{cM}$ intervals or less could be tried. Sibpair or affected pedigree member analysis 


\begin{tabular}{|c|c|c|c|c|c|}
\hline & \multicolumn{2}{|c|}{ Prediabetic state } & \multicolumn{3}{|c|}{ Diabetes mellitus (NIDDM) } \\
\hline & NGT Stage I & IGT Stage II & Preclinical Stage IIIa & Diagnosis Stage IIIb & Late diab. state Stage IIIb \\
\hline Fasting hyperglycemia & - & - & + & +++ & ++ \\
\hline Fasting hyperinsulinemia & + & $+(+)$ & ++ & ++ & $(+)$ \\
\hline Early insulin response to IVGTT & $+\rightarrow-$ & - & - & -- & -- \\
\hline Insulin resistance $(\downarrow R d)$ & + & $+(+)$ & ++ & +++ & +++ \\
\hline Dyslipidemia ( $\uparrow$ TG, $\downarrow$ HDL) & $(+) \rightarrow+$ & + & ++ & +++ & ++ \\
\hline Elevated plasma NEFA & $(+)$ & + & $+(+)$ & +++ & ++ \\
\hline Abdominal obesity & $(+) \rightarrow+$ & + & ++ & +++ & ++ \\
\hline Reduced VO2 max & + & + & ++ & ++ & ++ \\
\hline Arterial hypertension & $(+)$ & + & + & ++ & +++ \\
\hline Macroangiopathy & $?$ & + & + & ++ & +++ \\
\hline Microalbuminuria & $(+)$ & + & + & ++ & +++ \\
\hline Proteinuria (nephropathy) & - & - & $(+)$ & + & $+(+)$ \\
\hline Retinopathy & - & - & + & $+(+)$ & +++ \\
\hline
\end{tabular}

NGT, Normal oral glucose tolerance; IGT, Impaired oral glucose tolerance.

has the advantage of no assumptions; one only compares the observed and expected frequency of a genotype in the affected members. If one decides to include unaffected siblings, knowledge of the metabolic phenotype is mandatory. It may, however, not always be possible to distinguish between genetic and metabolic insulin resistance.

\section{The thrifty phenotype theory}

It has recently been suggested that intrauterine nutrition may play a major role for the development of subsequent diabetes (30). In a study from England, birth weight of patients with NIDDM was significantly lower than the birth weight of agematched controls. Children with a low birth weight also developed other signs of the insulin resistance syndrome later in life, such as hypertension, abdominal obesity, and high triglyceride concentrations. On the other hand, a high birthweight is also associated with an increased risk for NIDDM later in life. The Phoenix group recently established the presence of a U-shaped curve related to the risk of birth weight in Pima Indians. They also estimated that a low birth weight contributes at most $5 \%$ to the overall risk of NIDDM in Pima Indians. However, it has to be kept in mind that maternal transmission seems to predominate in NIDDM. Imprinting of maternal (or paternal) genes could explain this apparent paradox, also, possibly the thrifty phenotype if genetic imprinting would ensue as a consequence of limited nutrient supply during gestation. In fact, a recent paper reported imprinting of paternal insulin genes in the yolk sac of mouse (31).

\section{The compensation theory}

Evaluated at their ambient fasting glucose level the glucose turnover rate is normal or near normal in both prediabetic and diabetic subjects (32). It means that these subjects are able to compensate for the reduced insulin-mediated glycogenesis in skeletal muscles. In the normoglycemic prediabetic state insulin resistance is compensated for by hyperinsulinemia. When this compensation fails hyperglycemia develops and hyperglycemia itself compensates for reduced insulin mediated glucose uptake. This ability to compensate for an inherited defect may explain why subjects with a putative genetic defect in glycogenesis have survived during evolution. However, the price they pay is hyperinsulinemia and/or hyperglycemia which leads to macroangiopathy. This compensation may link insulin resistance to the increased morbidity and mortality seen in subjects suffering from "the insulin resistance syndrome."

\section{Prediabetic and diabetic stages of NIDDM}

NIDDM starts much earlier than we thought so far. Diabetes rather represents the end of the spectrum, "the top of the iceberg," and at this stage many patients already have developed all the macroangiopathic complications typical for the disease. The metabolic derangements (insulin resistance and compensatory hyperinsulinemia) responsible for the NIDDM syndrome can probably be demonstrated, decades before the disease is diagnosed. The hyperglycemic state itself may start $10 \mathrm{yr}$ before the clinical presentation of diabetes (3), which may influence progression of both micro- and macroangiopathy. At diagnosis of NIDDM $\sim 40 \%$ of patients already present macroangiopathy, $40 \%$ present micro- or macroalbuminuria, $15 \%$ present retinopathy, $50 \%$ are hypertensive, and $\sim 50 \%$ suffer from hypertriglyceridemia $(2,33)$. Before the hyperglycemic preclinical stage a long period of normoglycemia, with insulin resistance and compensatory hyperinsulinemia, is observed. This period may be divided into a stage of normal (NGT) and impaired glucose tolerance (IGT) that always precedes diabetes. Each period is phenotypically characterized by different degrees of metabolic and morphological abnormalities. Based on that we propose the following staging system for "the NIDDM syndrome"' (Table I).

Stage I represents the prediabetic stage characterized by NGT but insulin resistance and varying degrees of fasting hyperinsulinemia. First phase insulin secretion response to iv glucose may be invariably increased in the first decennials. Abdominal obesity, reduced $\mathrm{V}_{\mathrm{O} 2 \max }$, microalbuminuria, and arterial hypertension may already be present at this stage (2). Stage II is the prediabetic glucose intolerant stage (IGT) characterized by insulin resistance, fasting hyperinsulinemia, but inappropriate insulin response to iv glucose. During this stage macroangiopathy is a common feature resulting in an excess mortality (2, 33). Stage III is the fulminant diabetic state. This state may be 


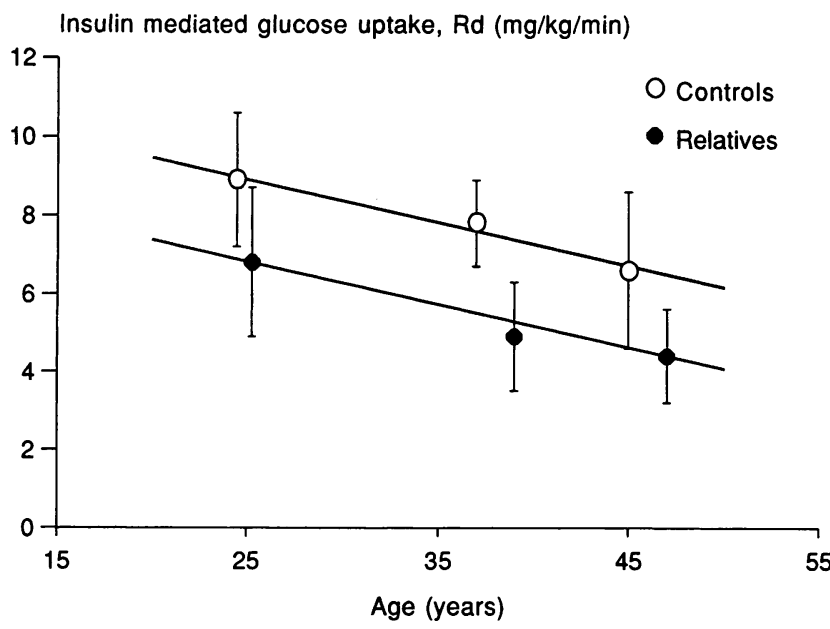

Figure 3. Insulin-mediated glucose uptake $\left(R_{d}\right)$ during a $40 \mathrm{mU} / \mathrm{m}^{2}$ per min clamp in controls and in relatives of NIDDM patients as the function of age $(20-50 \mathrm{yr})$. Values above the upper line are considered to be normal, whereas values below the lower line are considered to be abnormal, i.e., insulin resistance. Values between the two lines may belong to both the normal and the relative population. The values given in this figure are based on the result of the three separate studies outlined in references $4,5,7$.

subdivided in the preclinical stage III a, during which diabetics are unaware of their disease, and in the clinical stage III b, starting with the diagnosis of hyperglycemia. We propose this staging system (which may fit into the ICD system by WHO) to be used both epidemiologically and clinically, but especially in prevention of the fulminant NIDDM syndrome. However, to use this system one may be able to characterize insulin-resistant subjects and therefore the question can be raised: is it possible to separate insulin resistant young subjects from subjects without risk for development of NIDDM? Until now only three studies of insulin action in young ( $<50 \mathrm{yr}$ of age ) first degree relatives of NIDDM patients have been published $(4,5,7)$. The results show that glucose disposal ( $R_{d}$ values) obtained at an insulin infusion rate of $40 \mathrm{mU} / \mathrm{m}^{2}$ per min is reduced (by $30 \%$ ) in all three studies, i.e., as a mean $5.6 \pm 1.3$ vs. $7.9 \pm 1.4$ $\mathrm{mg} \times \mathrm{kg} / \mathrm{min}$. (Fig. 3). It means that relatives to NIDDM patients with $R_{d}$ values below the mean value did not overlap with controls (mean value minus $1 \mathrm{SD}$ ). In Fig. $3 R_{d}$ values are plotted against age for both controls and relatives. Obviously, $\mathbf{R}_{d}$ values expressed per $\mathrm{kg}$ body weight are decreasing with age since the relationship is linear. Based on these data all values below the lowest line may be considered as abnormal (66\% confidence interval), i.e., representing insulin resistance. Fig. 3, therefore, can be used for classification of prediabetic subjects.

\section{Prevention of NIDDM}

This staging system could help to improve diagnosis and treatment of the NIDDM syndrome, but also to provide the clinician with the necessary tool to prevent progression. This division into clinical and infinitive stages may of course be arbitrary and imprecise, but may also, as stated by others (3), help us to understand the NIDDM syndrome metabolically, genetically, and clinically. The history of treatment of NIDDM has been a history of failures. This is not surprising considering how far advanced macroangiopathic complications are at diagnosis of the disease $(2,32)$. Therefore, prevention of NIDDM and its macro- and microvascular complications represent the real challenge for our health care system!

If a subject fulfills all the criteria for stage I of the NIDDM, syndrome prevention of diabetes may be considered depending on age and other risk factors as weight, arterial hypertension, hypertriglyceridemia, and microalbuminuria. If the subject belongs to stage II, prevention seems obligate since most of these subjects evidently develop frank diabetes and present overmorbidity and overmortality compared with controls (2).

But how can NIDDM be prevented? If we accept that NIDDM is a result of genetic predisposition exposed to an imbalance between energy intake and expenditure, we have some options. It may take some time before the genetic predisposition is unraveled. In the meantime, we can recommend the patients to reduce energy intake or increase expenditure, i.e., performing physical exercise in order to avoid obesity. An increase in energy expenditure and/or a reduction of energy intake has been proven in prospective studies to prevent development of NIDDM and reduce mortality in prediabetic subjects $(34,35)$.

\section{Acknowledgments}

Inger Riegels, Anette Steffensen, and Ulla Wetterlundh are thanked for help with the preparation of the manuscript.

\section{References}

1. Reaven, G. M. 1988. Role of insulin resistance in human disease. Diabetes. 37:1595-1607.

2. Harris, M. I., W. C. Hadden, W. C. Knowler, and P. H. Bennett PH. 1987. Prevalence of diabetes and impaired glucose tolerance and plasma glucose levels in U.S. populations aged 20-74 yr. Diabetes. 36:523-34.

3. Granner, D. K., and R. M. O'Brien. 1992. Molecular Physiology and Genetics of NIDDM. Diabetes Care. 15:369-388.

4. Eriksson, J., A. Franssila-Kallunki, A. Ekstrand, C. Saloranta, E. Widen, C. Schalin, and L. Groop. 1989. Early metabolic defects in persons at increased risk for non-insulin dependent diabetes mellitus. N. Engl. J. Med. 321:337-343.

5. Vaag, A., J. E. Henriksen, and H. Beck-Nielsen. 1992. Decreased insulin activation of glycogen synthase in skeletal muscles in young nonobese Caucasian first-degree relatives of patients with non-insulin-dependent diabetes mellitus. $J$. Clin. Invest. 89:782-788.

6. Martin, B. C., J. H. Warram, A. S. Krolewski, R. N. Bergman, J. S. Soeldner, and C. R. Kahn. 1992. Role of glucose and insulin resistance in development of type 2 diabetes mellitus: results of a 25-year follow-up study. Lancet. 340:92529.

7. Gulli, G., E. T. L. Ferrannini, M. Stern, S. Haffner, and R. DeFronzo. 1992. The metabolic profile of NIDDM is fully established in glucose-tolerant offspring of two Mexican-American NIDDM parents. Diabetes. 41:1575-1587.

8. Vaag, A., J. E. Henriksen, N. Holm, and H. Beck-Nielsen. 1993. Insulin secretion, peripheral insulin sensitivity and hepatic glucose production (HGP) in identical twins discordant for NIDDM. Diabetes. 42, suppl. 1:71A.

9. Skarfors, E. T., K. I. Selenus, and H. O. Lithell. 1991. Risk factors for developing non-insulin dependent diabetes mellitus: a 10-year follow-up of men in Uppsala. Br. Med. J. 303:755-760.

10. Eriksson, K. F. 1992. Prevention of non-insulin dependent diabetes mellitus. Akademisk Avhandling, Lunds Universitet.

11. Hother-Nielsen, O., and H. Beck-Nielsen. 1991. Basal glucose metabolism in type 2 diabetes. A critical review. Diabete \& Metab. 17:136-145.

12. Taylor, S. I., and A. Accili. 1991. Molecular genetics of insulin resistant diabetes mellitus. J. Clin. Endocrinol. Metab. 73:1158-1163.

13. Freidenberg, G. R., D. Reichart, J. M. Olefsky, and R. R. Henry. 1991. Reversibility of defective adipocyte insulin receptor kinase activity in non-insulindependent diabetes mellitus: effect of weight loss. J. Clin. Invest. 88:1323-1330.

14. Almind, K., C. Bjoerbaek, H. Vestergaard, T. Hansen, S. Echwald, and O. Pedersen. 1993. Aminoacid polymorphism of insulin receptor substrate-1 in non-insulin dependent diabetes mellitus. Lancet. 342:828-832.

15. Reynet, C., and C. R. Kahn. 1993. Rad: A member of the Ras family overexpressed in muscle of Type II diabetic humans. Science (Wash. DC). 262:1441-1444.

16. Schalin-Jäntti, C., H. Yki-Järvinen, L. Koranyi, R. Bourey, J. Lindström, P. Nikula-Ijäs, A. Franssila-Kallunki, and L. C. Groop. 1994. Effect of insulin on 
GLUT-4mRNA and protein concentrations in skeletal muscle of patients with NIDDM and their first-degree relatives. Diabetologia. 37:401-407.

17. Bell, G. I., K. S. Xiang, and M. V. Newman MV. 1991. Gene for noninsulin dependent diabetes mellitus (maturity-onset diabetes of the young) is linked to DNA polymorphism of human chromosome 20q. Proc. Natl. Acad. Sci. USA. 88:1484-1488.

18. Lehto, M., K. Xiang, M. Stoffel, III R. Espinosa, L. Groop, M. M Le Beau, and G. Bell. 1994. Human hexokinase II: Localization of the polymorphic gene to chromosome 2. Diabetologia. 36:1299-1302.

19. Damsbo, P., A. Vaag, O. Hother-Nielsen, and H. Beck-Nielsen. 1991. Reduced glycogen synthase activity in skeletal muscle from obese patients with and without Type 2 (non-insulin dependent) diabetes mellitus. Diabetologia. 34:239-245.

20. Wells, A. M., I. C. Sutcliffe, A. B. Johnson, and R. Taylor. 1993. Abnormal activation of glycogen synthesis in fibroblasts from NIDDM subjects. Evidence for an abnormality specific to glucose metabolism. Diabetes. 42:583-589.

21. Groop, L., M. Kankuri, C. Schalin-Jäntti, A. Kestrand, P. Nikula-Ijas, E. Widen, E. Kuismanen, J. Eriksson, A. Franssisla-Kallunki, C. Saloranta, and S. Koskimies. 1993. Association between polymorphism of the glycogen synthase gene and non-insulin dependent diabetes mellitus. N. Engl. J. Med. 328:10-14.

22. Lehto, M., M. Stoffel, L. Groop, R. Espinosa III, M. M. Le Beau, and G. I. Bell. 1993. Assignment of the gene encoding glycogen synthase (GYS) to human chromosome 19, band q 13.3 Genomics. 15:460-461.

23. Orho, M., X. Huang, M. Lehto, C. Schalin-Jäntti, P. Nikula-Ijäs, and L. Groop. 1994. Polymorphism of the glycogen synthase gene (GS) and other markers in patients with NIDDM. Diabetes. 43(Suppl. 1):13a. (Abstr.)

24. Vestergaard, H., S. Lund, F. S. Larsen, O. Bjerrum, and O. Pedersen. 1993. Glycogen synthase and phosphofructokinase protein and mRNA levels in skeletal muscle from insulin-resistant patients with non-insulin dependent diabetes mellitus. J. Clin. Invest. 91:2342-2350.

25. Froguel, P., M. Vaxillaire, and F. Sun. 1992. Close linkage of glucokinase locus on chromosome $7 \mathrm{p}$ to early-onset non-insulin dependent diabetes mellitus. Nature (Lond.). 36:162-164.

26. van den Ouwenland, J. M. W., H. H. P. J. Lemkes, and W. Ruitenbeek. 1992. Mutation in mitochondrial tNA ${ }^{\text {Leu(UUR) }}$ gene in a large pedigree with maternally transmitted type 2 diabetes mellitus and deafness. Nature Genet. 1:368371 .

27. Neel, V. 1962. In Diabetes mellitus: a "thrifty" genotype rendered detrimental by progress? Am. J. Hum. Genet. 14:353-362.

28. Coleman, D. L. 1979. Obesity genes: beneficial effects in heterozygous mice. Science (Wash. DC). 203:663-665.

29. Seldin, M. F., D. Mott, D. Bhat, A. Petro, C. M. Kuhn, S. F. Kingsmore, C. Bogardus, E. Opara, M. N. Feinglos, and R. S. Surwitt. 1994. Glycogen synthase: a putative locus for diet-induced hyperglycemia. J. Clin. Invest. 94:269276.

30. Hales, C. N., and D. J. P. Barker. 1992. Type 2 (non-insulin-dependent) diabetes mellitus: the thrifty phenotype hypothesis. Diabetologia. 35:595-601.

31. Giddings, S. J., C. D. King, K. W. Harman, J. F. Flood, and L. R. Carnaaghi. 1994. Allele specific inactivation of insulin 1 and 2, in the mouse yolk sac, indicates imprinting. Nature Genet. 6:310.

32. Vaag, A., P. Damsbo, O. Hother-Nielsen, and H. Beck-Nielsen. 1992. Hyperglycemia compensates for the defects in insulin mediated glucose metabolism and in the activation of glycogen synthase in skeletal muscles in patients with Type 2 (non-insulin-dependent) diabetes mellitus. Diabetologia. 35:80-89.

33. Olivarius, N.dF. 1992. Diabetic complications presenting in 1360 newly diagnosed middle-aged and elderly diabetics. 13th WOWCA World Conference on Family Medicine (Abstract).

34. Helmrich, S. P., D. R. Ragland, R. W. Leung, R. Paffenbarger. 1991. Physical activity and reduces occurrence of non-insulin-dependent diabetes mellitus. N. Engl. J. Med. 325:147-52.

35. Eriksson, K. F., and F. Lindgärde. 1991. Prevention of Type 2 (noninsulin-dependent) diabetes mellitus by diet and physical exercise. Diabetologia. 34:891-898. 